\title{
Towards Adaptive user Interfaces for Mobile-Phone in Smart World
}

\author{
Muhammad Waseem Iqbal ${ }^{1}$, Nadeem $\mathrm{Ahmad}^{2}$, Syed Khuram Shahzad ${ }^{3}$, Irum Feroz ${ }^{4}$, Natash Ali Mian \\ ${ }_{1,2,3,4}$ Department of Computer Science \& IT \\ The Superior College (University Campus) \\ Lahore, Pakistan \\ ${ }^{5}$ Beaconhouse National University \\ Lahore, Pakistan
}

\begin{abstract}
All applications are developed for context adaptation and provide communication with users through their interfaces. These applications offer new opportunities for developers as well as users by collecting context data and adapting systems behavior accordingly. Particularly, in mobile devices, these mechanisms provide usability increment tremendously. Rigid and non-adaptive interface blocks the features of context awareness. In this paper, we study methods, technologies and criteria which have been proposed specifically for adaptive interfaces. Based on these guidelines, we elaborate the intelligence of adaptivity and usage of context according to user mental model. Further, we have proposed a model to develop user context ontology (UCO) and adaptive interface ontology (AIO) to optimize the use of adaptive mobile interfaces in the context of user preferences. These ontologies organize the perceptions and thoughts of user. The philosophy of User Centered Design (UCD) is proposed to analyze the usability and validity of mobile device interfaces according to user contexts.
\end{abstract}

Keywords-Adaptive features; smart-phone; usability experience, user interface; user context; usability engineering; UCD

\section{INTRODUCTION}

The mobile-phone interfaces provide contented and dependable communication medium between the device and the user. Currently, mobile phones present interfaces having various styles and modes of interaction. These interfaces have different usability requirements and measures, that make the process of interaction design more complex [1]. The competitive environment has used the burst of technical options in interaction design [2] in which interface developers and end users face more difficulties in understanding of hardware and software technical details. Computer interfaces are emerging to be more sovereign in functionality, software systems are getting more complex, and online information spaces are growing rigorously in size. With tremendous growth in technical support, the variant usage patterns are also emerging [3]. There is now immense number of new users, who are not technology expert, but rather naïve to computing devices such as non-technical professionals, elderly people, and children [4]. These users have not only diverse computer skills, but they vary in many other aspects like their knowledge, skills, intellectual and physical capabilities, mood, motivation and most importantly the target tasks and usage of technology.
Numerous high-quality applications introduced but missout from the market [5] due to their complex, unattractive, inefficient and confusing user interfaces. The impact of nonadaptive user interface for mobile devices creates frustration and directly effects on performance, usability and reception amongst the users. During the designing of mobile phone interfaces [6], the user requirements should be evaluated in terms of usability, learnability, understandability, effectiveness, efficiency and objectivity. Latest mobile operating systems like Android or iOS provide kids, guest, driving and night modes for the accessibility of applications according to the user's task. These interaction modes are provided for specialized context with variation in values of common features like daylight, age groups and access styles. These modes provide pre-defined and static profiles [7] with factory settings. The user context study and analysis are still missing in the interface settings for predefined interaction modes. User is bound to select the most suitable yet rigid modes in any other context.

Adaptive User Interfaces (AUIs) can provide a lot of benefits to address these usability concerns. User interface adaptation has been identified, quite a while ago, as an imperative subject to address in modern information systems design. There have been three adaption design techniques for three major aspects of adaption. The information adaptation selects information to present. The presentation adaptation states the presentation styles of this information. The last interface adaptation defines the interaction mode and style [8]. With these questions answered at design level, the adaptation of mobile user interfaces provides big support for the user satisfaction.

The heterogeneity in user physical demographic properties and limitations of smart device interaction style brings us a challenge to develop a specialized interface for variety of users [9] [10]. Many users face problems in customization panels. These setting dialogues are very difficult to understand in formation especially for users with disabilities and lesser ICT knowledge and skills. Similarly, the need for customization is a significant requirement demanded by children or elderly people. Hence, customization of any device that targets accessibility must include auto-adaptation and self-learning mechanisms for user's requirements. Bad user interfaces and usability disorientation may cause annoyance and could lead to unsatisfied behavior amongst users [11]. For effective mobile interfaces the intended tasks should be mapped with user's 
mental model [8] [12]. Currently, the benefits of AUI have been realized to develop the interfaces in user's context. The AUI is a feasible adaptation approach than adaptable, because it provides suitable methodology of adaptation and it can handle the usability issues of user's interaction [13] [14]. The adaptive user interfaces are proposed as solution to cater the problems that enable any mobile application to provide dynamically customized interface [5] for different groups of users having similar properties and needs. The user's contextbased interfaces help to make the collaborative, supportive, constructive and communicative activities easy.

The research idea under discussion shows the User Centered Design (UCD) approach to add adaptivity in mobile device interfaces. Therefore, the implementation of mobile adaptive user interfaces is very necessary for user's learnability, efficiency and satisfaction. The adaptive interfaces, designed according to the user requirements will help to enhance the user interaction with mobile devices.

\section{ADAPTIVITY AND ADAPTABILITY IN MOBILE-PHONE USER INTERFACE}

The two approaches such as adaptivity and adaptability are used to personalization for user interfaces of mobile devices. The aim of both interfaces is to provide personalization for the users while these two approaches are different in the adaptation process [15]. Recently, the adaptivity and adaptability have achieved high popularity on the world wide web (WWW) under the notion of personalization [16]. The reason of this popularity is due to the less homogeneity of website users than the users of commercial software's.

In adaptive approach, the interface automatically adjusts and assists the user. The adaptive user interfaces can adapt their activities by monitoring user status, the state of system and the current situation according to adaptation strategy. Usually the intensity of adaptation is measured in the case of effectiveness, efficiency and satisfaction for user interface [4] [17]. The factors such as spatial stability which increases the user satisfaction and high locality, improves discoverability of adaptation in representing interface in its original position. Spatial stability is required to maintain the mental model of application. Moreover, accuracy provides the user perception for algorithm predictability, whereas the user interfaces with higher accuracy provide more predictability and consistency. Further, the interaction frequency and task complexity play an important role in the perception of adaptation [6]. If there is a need of large mechanical interactions with simple tasks [18], the adaptation locality plays important role for complex tasks. The users are able to create mental models for applications to interact frequently with low complexity.

Conversely, the adaptable interfaces provide mechanisms of customization but rely on the adaptation of user mechanisms. In adaptability, the user's preferences and characteristics are known before creating interaction [19]. The information system can be adopted manually by the user or administrator or automatically by the system to fulfill the requirements of users. AVANTI system provided adaptivity and adaptability features within the user interface. This system provides special input and output devices, visual/non-visual interface objects and integrated interaction techniques. The contents, modality and prominence of information, navigation aids, search facility and links to other hypermedia pages are adapted [20] [21]. One of the advantages of adaptable systems is that the users are completely controlled through the interface. On the other side, the behavior of adaptive systems is completely matched with user's mental model. It provides interaction with systems by considering the user performance, ease of system, minimizing request help, removing complexity and avoiding the problems of cognitive overload [14]. Adaptations always support to achieve goals of users for performing actual tasks rather than incorrect predictions [22]. However, considerable amount of work needs to be performed on user's side to adapt the interface. Table 1 elaborates the difference in adaptive and adaptable interfaces.

TABLE I. COMPARISON BETWEEN ADAPTIVE AND ADAPTABLE SYSTEMS

\begin{tabular}{|l|l|l|}
\hline & Adaptive & Adaptable \\
\hline Definition & $\begin{array}{l}\text { It has dynamic adaptation by } \\
\text { the system itself to perform } \\
\text { the current task. }\end{array}$ & $\begin{array}{l}\text { In adaptable system, the user } \\
\text { can change functionality of } \\
\text { the system. }\end{array}$ \\
\hline Knowledge & $\begin{array}{l}\text { It is contained within a } \\
\text { system. }\end{array}$ & It is extended. \\
\hline Strengths & $\begin{array}{l}\text { There is no need of special } \\
\text { effort and knowledge by the } \\
\text { user. }\end{array}$ & $\begin{array}{l}\text { The system has control of } \\
\text { user. Also, user knows the } \\
\text { task which is needed to } \\
\text { successfully completed. }\end{array}$ \\
\hline Weaknesses & $\begin{array}{l}\text { There are few success models } \\
\text { exists. User has difficulty to } \\
\text { develop an intelligible system. }\end{array}$ & $\begin{array}{l}\text { System complexity and } \\
\text { incompatibility increased. } \\
\text { User needs to learn adaptation } \\
\text { component. }\end{array}$ \\
\hline $\begin{array}{l}\text { Mechanism } \\
\text { Required }\end{array}$ & $\begin{array}{l}\text { Models of users, tasks, and } \\
\text { dialogs; knowledge base of } \\
\text { goals and plans; powerful } \\
\text { matching capabilities; } \\
\text { incremental update of models. }\end{array}$ & $\begin{array}{l}\text { Layered architecture; domain } \\
\text { models and domain- } \\
\text { orientation; "back-talk" from } \\
\text { the system; design rationale. }\end{array}$ \\
\hline $\begin{array}{l}\text { Application } \\
\text { Domain }\end{array}$ & $\begin{array}{l}\text { Customization, differential } \\
\text { descriptions and information } \\
\text { retrieval system is required. }\end{array}$ & $\begin{array}{l}\text { Information retrieval, end-user } \\
\text { modifiability, tailor ability, } \\
\text { altering, and design is used. }\end{array}$ \\
\hline
\end{tabular}

The graphical user interface mechanism to control the customization is usually provided by non-programmatic customizable systems [23] [24]. Generally, this type of interfaces is very helpful for providing direct manipulation of interfaces. The user satisfaction level over the interface interaction can be increased by using this type of customization. It also provides the sense of achievement about completion of tasks especially in situations where responsibility needs to be assigned to the user of a critical system.

\section{USABILITY OF MOBILE-PHONEINTERFACE}

Usability of interfaces for mobile phone devices is really concerned with the satisfaction of users. The basic purpose of usability is to provide guidance for developers to develop user friendly applications. It is not easy to define and evaluate the usability as formal or informal specific environment. Still there is not a precise apparatus to measure the absolute usability of 
any product [25]. Currently, the term usability is used to enhance interactions among users for their products to complete the tasks according to the environment [26]. In human computer interaction (HCI) the usability [27] is considered as one of the major concepts which has already produced emerging views regarding product usage and customer's satisfaction [28]. The Jakob Nielson [29] is one of the pioneers who tried to objectively evaluate the user experience on digital platforms. Though, it dated back to the 90s when he defined ten important usability heuristic principles (visibility of system status, match between system and real world, user control and freedom, consistency and standards, error prevention, recognition rather than call, flexibility and efficiency of use, aesthetic and minimalist design, help users recognize, diagnose and recover from errors, help and documentation) which are still valid and used in everyday life [30]. Moreover, different attributes of usability (e.g. efficiency, learnability, memorability, rate of errors and satisfaction) are also available for consideration when designing a product or interface. If the interface of any product or mobile phone is difficult to use, even it has excellent functionalities, it doesn't matter, the user will shift towards easy alternative [31]. During the analysis phase of software development, the usability is considered very important for user satisfaction in which user needs to interact with the system. Another aspect is usability architectural impact, which is not only concerned with system's outlook but also required the better user experience (e.g. undo, redo, cancel and selection etc.). By considering the complexity of mobile phone interface usability, there are multiple solutions that have been proposed through user centered methodologies. Amongst the various existing user models, there is not an integral model available which considers the different complex features such as cognitive, motor and psychological [32]. Throughout the usability evaluation, the complexity of major issues is found more multifaceted rather than minor issues. Hence, more effort is required to identify the problems which are violating the heuristics such as missing elements in user interface are difficult to check during interface evaluation. Nielsen [33] evaluated the same product that contained different marked problems. The usability experiments of software application for new users took individually and were also conducted in team. The only one problem was identified as common in four teams while others were quite different.

Molich et al. [34] [35] evaluated the same website of nine organizations to measure the consistency in usability testing. As a result, 310 total problems were reported and 232 of them were unique. Efficiency, effectiveness and satisfaction are the parameters of usability in ISO 9241-11 standard. Although this standard is very compact but provides minor discussion about evaluation of usability. This standard also gives comparatively limited guidelines about how to interpret scores from specific usability metrics.

\section{A. Usability of Mobile-Phone Operating Systems}

Recently the usage of mobile phone devices is increasing dramatically throughout the world. There are two major vendors (iOS and Android) prominently available in the global market. iOS is projecting choice of users by providing friendly GUI. It (Apple) also provides a leisure time to customers with most trusted hardware support. On the other hand, Android is also a prominent brand but still struggling for better hardware support. Along with hardware, the usability of mobile phone applications is becoming an increasingly significant part amongst users [36]. Moreover, the operating systems (OS) along with technical possibilities and application compatibilities to enable the functionalities of end device can play vital role in terms of task performing [37]. To measure the usability of smart phone applications, tests were taken on the basis of Ericsson and Simon's work by using think aloud protocol. This protocol encourages the users to think loudly while performing their specific tasks. On the collected information, the user's interface promotes the natural human thinking capabilities to enhance the performance and improvement [38]. The features of interface are developed differently in separate devices according to user desires for better usability. These interfaces may provide large monitors, small screens, enhanced input/output devices (e.g. trackball, keyboard or touch pen) to improve the efficiency, effectiveness and satisfaction [39] [40]. The mobile phone user interfaces should be developed to accommodate the users according to their context.

\section{Mobile USAGE CONTEXT}

An interesting point of view about context states that it includes the user's state of emotions, focal point of user's attention, demographics and all the elements present in the user's environment [41]. Even the term context in vocabulary refers to the environment of usage providing details about the user's technological knowledge, user's current state of affairs and application's background, application settings and features of the current usage situation [42] [43]. Thus, it highlights the importance of precise definition of all information that should be considered as context for adaptive application development of mobile phones.

With the above-mentioned aim, we deduced the following major context entities for device, task and usage learning. Usage learning context aims to explore and state the user properties and preference that are quite important instrument for proper adaptations application. In scenario of mobile applications, the user model can be useful in several conducts: the user historical usage pattern can identify and even predict the user needs and select information of use's interest [44]. Such information present principally a significant positive impact on application's interface and its proposed content. The task to model the user with a mobile device and varying environment appears to be much difficult than performing the same for a user in desktop environments [45]. Usability is mostly coupled with interface only while it is the property of overall system. It refers to the quality of use in a specific context [46]. As mentioned above, current methods and techniques for usability prediction are inadequate regarding their accuracy due to consideration of partial context of users, tasks and environment.

There exists a lot of methods to determine the quality of use of any system especially for ICT domain. These evaluations include the effectiveness measures of system by calculating the successful achievement of user's intended goals. The efficiency of the system in measured over the required 
resources to achieve user's goal. The resource list may include time, money or user's mental effort. The satisfaction is judged over the acceptability of overall system by the user [4]. The overall picture of a system presents users, tasks and technical resources (hardware, software and materials) of that systems surrounded by the physical and organizational infrastructure. All of these elements of the system environment greatly influence the interaction. The promotional campaigns, like "Usability Now!" in the UK, have brought awareness in the buyers of any system about the importance of usability. With the effect of these awareness programs, buyers now give more consideration to ease of use of any (S/W or H/W) systemin their selection. Similarly, the producers and suppliers of the product with high usability get customers attention and a market edge. Microsoft and Amstrad have highlighted the ease of use as a major selling feature in their recent advertising campaigns and gain sales promotions [47]. International usability and the user interface standards are being increasingly referred in public procurement. They also aim to fulfil European Display Screen Equipment Directive.

\section{A. Context Properties}

Context-aware computing methods and techniques have been used in most of the research for adaptive interface development. The said methodology comprises of sensor technology to collect information about surrounding environment like location, time, daylight, temperature, user identity and action. Adaption with more details has not been generally adopted for such systems like, data input methods based on context. In this research, we tried to identify the user's properties and behavior using domain experience and mobile technology experience. It also aims to congregate all the context elements with scale and value ranges that influence the user's task performance. Consequently, provide design guidelines for the interfaces that can automatically adapt according to the provided context information [48]. The user itself, as a compound entity, is an essential part of the context. There has been very specialized set of behaviors provided by any context-aware application to react against specific context variations. Thus, the software engineer must clearly understand the development goals and classify various context cases in the targeted application.

1) User properties: User's properties are most concerned in many of the context aware applications where context in represented by the user's status like user's age, gender, demographies and emotions. User's demographical properties like location, time, weather or activities are usually recognized through internal/external sensors (e.g.: GPS, accelerometer, Web data camera, microphone), while the emotional state can be mined from user's current responses in contrast with the history of user's actions [49]. The classification and categorization of user's properties is unlike to a data ontology of a person used in social network. User properties can be categorized over the semantics and use of the values in the universe of discourse. Some of the user properties are brought up for user's identification like name any IDs (username, social security number), street, address, city, country, address properties (zip code, country code, telephone number, network code, phone number, home address and email are part of contact details and other business properties may include date of birth) [50]. As humans have diverse lifestyle requirements and these aspects should be modeled through user profile modeling. The profiling of the usercan be used for the desired level of personalized service delivery which attains the capability to adapt itself for a particular user. The user profile is a digital representation of the user and context-aware system places it in the modeling and management layer.A user profile can be characterised as a number of user-related classes. Various dynamic profile aspects have been discussed in five profile classes consisting of CapabilityProfile, InterestProfile, PreferenceProfile, EducationProfile and HealthProfile [51]. Needs of user with specialized requirements have also been addressed in an ontology engineered for user profile to assist people with dementia in mobile usage environment.

2) Device properties: Device context is directly influencial to the usability of any application. Any interface neglecting the device properties cannot achieve ease of use. Incorporation of device elements in interface design process become more severe and complex with the diversity of mobile devices introduced. A mobile application has to be compatible,rather comfortable with several versions. No doubt, currently, mobile devices competencies fulfill many tasks according to user's requirement with multiple hardware options. Since each device claims its own individuality, it is not trivial to design and develop a mobile application. As a mobile application developer, we can refer to the operating system on which this application will be deployed and run while using all hardware facilties (e.g. network connectivity, display, GPS sensors). All these device properties play a vital role mobile applications development. The device context is, in general, considered in the designing and development of mobile application interfaces that works on various operating systems and hardware platforms. The development frameworks, provided by different OS platform association like android or iOS, suggest the functionalities to read the technical information of the device (e.g. memory, display size and resolution, list of sensors and network connectivity options) [52]. The device properties may include some of the followings:

- Font size (Small, medium, large)

- Font color (RGB color, black \& white)

- Font format (Times New Roman, Tahoma, etc.)

- Background color (Auto adjust, changing manually)

- Data entry mode (Typing, tapping, voice)

- Display information (Text, sound, LED lights)

- Message delivery (Text, voice, alert, silent, pre-answer)

- Brightness level (Increase/decrease)

- $\quad$ Ring volume (Low, medium, high, alert, vibration)

- Sound level (Mute, regular, loud) 
3) Environmental properties: Environemtal properties presents the information about the surrounding environment like spatio-temporal information. The time and space knowledge can be gathered through some sensors with collective knowledge of the cloud. The time can be scaled in various ways like daylight time, office timing, hours/minutes/second, weekend/weekday etc. While space properties may include the location and other properties of surrounding location like weather, humidity, luminosity or noise level. In literature, Module6 of CoDAMoS ontology has been reused for environment modeling in context ontology. Thus, we got a nucleus model for environments and their semantic relationships with the above stated environmental conditions [53] [54]. Different domains of the mobility environment are experts in mobility infrastructure, service development, device connectivity and interface design, etc. [52]. The mobility of the devices tranfering from one to another environment makes it essential to consider the usage environemt in mobile application interface development.

4) Tasks properties: The usability of a mobile application also depends on the task performed and the interface supporting specific tasks. Adaptivity features of a mobile application according to the current activity of user presents another type of context consideration in mobile application designing and development. Unlike the type of context discussed earlier that sense the physical properties, task properties may organise the user interface, with learning the aim of use. The adaptable modalities (e.g. voice, speech synthesis, device vibrations, gesture) are used as a communication way with respect to the tasks between the user and the application [4]. During any adaptive application development, developers need to identify the intent and activities. It is also related to the functionalities provided in specific context by the mobile application. In this way [55], the application is required to read the environment to determine the current activity to be performed by the user. Here, the designer need some state identification to analyze the task and decide the communication options (e.g. sensors, touch screen, network). Another question that arises is to select the proper sensing device or software to determine the intended actions. The option of profile selection (e.g. driving, sport, night) can be given for a specific activity (e.g. texting, calling or listening to news etc.) [56]. Task properties play important role in the development of adaptive features. The context of user activity is necessary to map with device behaviour to fulfill the user desires.

\section{B. Special Needs}

Specialized applications and some operating systems interface have been designed for the user with special need or any physical disorder. Assistance for the users with special needs enhance the overall usability of the systems that considers exceptional members of the target user range. These special needs may be provided for people with low and impaired vision due to age factor. Another colour vision problem is colour blindness that refers to a physical deficiency to identify and distinguish among some basic colours (i.e. red, green, blue) in the normal luminosity. These users having colour-blindness may be inadequate to differentiate within specific colour pairs. The applications developed for mobiles may avoid those specific pair of colours in various objects identification in one canvas especially in foreground and background combinations. It is also suggested to provide a colour transformation by the OS [57] to avoid such combination in application running at that platform.

Another study for Deaf' or better to be called naturally challenged users mostly suffer with great hearing loss. Sign Language (SL) can be used as the first language of communication for these naturally challenged users. There can also be options for such users to learn the Sign Language that is based on the combination of movements of hands, arms, body and facial expressions. A study [12] states that there has been nearly sixty thousand people living in Italy who use deaf Italian Sign Language (LIS) as their primary mean of communication.

\section{USABILITY ENGINEERING FOR MOBILE-PHONE}

Usability of smartphone applications is one of the major concerns in industry today. The trade-offs in user interfaces done by the manufacturers and application developers have resulted in dissatisfaction amongst the users. One of the major reasons is their non-contextual user interfaces [45]. AUIs help us in addressing these problems and increase the usability of applications by providing the functionality that focuses on the user requirements.

Variable regarding user's environment are available in wide range such as demography, cognitive skills, background, education, personality and preferences [46]. The interpretation of different users may not be matched for command names, icons and displays which is one of the major challenges in HCI. In smart world, there is a rapid increase in user interaction with interfaces and direct influence of the context on the user's task in an environment. The context and task define the change that needs to be performed at a specific movement on user's interface. In mobile computing, the context-awareness or physical environment includes surroundings of a user and device (e.g. location and time) [58] [59] [60]. User modeling, in the area of mobile applications can be performed by monitoring users' past behaviors or user profiles. This type of predictive knowledge has an influence on application interfaces and its contents. Context modeling for usage learning has been defined into three categories such as (i) based on domain knowledge (ii) supervised learning approaches and (iii) unsupervised learning approaches. Supervised learning approaches require smaller amount of domain knowledge while unsupervised learning do not require the domain knowledge [52] [61]. It is very difficult to collect relevant information from users because most of the users are not aware which information is important [41]. This issue may create difficulties for application developers to develop adaptive applications according to user's contextual information.

\section{A. Adaptivity and Intelligence in Mobile-Phone Interface}

The principles that lay the foundation of adaptive systems consider the situations that make the need of adaptation 
necessary. Based on these requirements appropriate adaptation plan is decided and the actions are taken accordingly. Fundamental choices that constitute the adaptation process of AUI design are [14]:

a) Establishing the role of the adaptation and by whom the adaptation will be performed.

b) Definition of overall goals of the user and the adaptation process.

c) Definition of adaptation rules which will manage the adaptation.

d) Definition of variables and level of interaction that is required for adaptation.

e) Definition of a complete inference mechanism and methods that will perform the adaptation process. All these methods should be in line with the user preferences.

The mobile phone industry is penetrating in the market and users need specialized interfaces to fulfill their requirements. Although adaptivity is the need of hour and it has some problems and tremendous benefits to create user satisfaction. HAoAok identifies [16] [62] a few problems of adaptive interfaces in one of his articles which summarizes the state of art in the field. First problem refers to the control of user where the user is not provided with the control of the adaptation, this is referred to as lack of control. Though the user involvement may increase the satisfaction of the user, but this may result in increasing the problems in developing the user model for adaptation. Second problem refers to the consequences of a user action. As user has no direct control over the adaptation process, hence the user is not sure about the results of some actions, this is classified as unpredictability. Third problem identified again refers to the user's understanding of the adaptation process and what the user actually expects from the interface. This makes it difficult for the developers to decide the portions that should be shown to the user at a given time, this problem is named as transparency. Fourth problem is termed as privacy, this means that user must accept the fact that everything that user is doing is recorded and will be used for adaptation of the user interface. On one side it is compulsory for the system to keep track of every task of user for building a profile, but, on the other side it creates a privacy concern for the user. Fifth problem refers to the trust on the adaptation process, according to literature; user's trust is volatile and may decrease further if the adaptation performed by the user does not fulfill his requirement.

AUI gives user flexibility of creation of interface at design level, not only this but it also allows the user to make the required changes during execution as well. This makes the user independent and does not restrict the developers and designers of the system to decide the optimal solution that are specific to a user. By using AUIs the system has the capability to adapt to the user need and also helps in future adaptations by keeping a history of adaptations performed [20] [58]. Adaptation has already been implemented in all types of systems ranging from desktop applications to web applications, smart phone applications, wearable device software and many more platforms.
The basic premise that an adaptive system work on is the user model, this model is built on the user's data, his behavior (which is recorded by keeping a track of actions by the user) and environmental conditions. The system predicts the user's activity and gives relevant information, functionality and suggestions to the user for the next task. By using this mechanism, the content is personalized for each user and is based on the user model developed by system at runtime [63], hence, the application performs differently for each user. This increases the user satisfaction as the feeling for using an application that understands an individual's preference is what every user wants. Another study analyzes the role of context in the telematics devices available in vehicles. Sixty-four students, including 35 male and 29 female students, from BenGurion University having average age of 25.7 participated in the study [58]. It was observed that the overall performance time decreased with the increase in adaptivity, hence the study concluded that adaptivity level directly effects the overall performance time of the user.

\section{B. Criteria to Develop Smart AUIs}

The need of user interfaces is rising gradually day by day. These interfaces are running on multiple devices along with various features. Any type of disability in interfaces creates motivation to develop the guidelines of interface generation and description of user interface. There are prominent approaches including MARIA, TERESA with Concur Task Trees, Personal Universal Controller (PUC) as used in Huddle and Uniform, UIML Canonical Abstract Prototypes (CAP) with recent modifications in CAP3. The following parameters may help to develop criteria for mobile phone adaptive user interfaces.

1) Run-Time adaptations and usability: Run time adaptation of user interface used in few systems to provide automatic generation and usability. However, numerous literatures have been written about potential problems of selfadaptive [64] user interfaces. Specially, it lacks in transparency which is described to improve the run time adaptations by animated transitions. Nevertheless, the aim of adaptive user interfaces to accessibility improvements may have a need of different approaches where the system takes a more proactive role [11]. In broader perspective, the goal of intelligent user interfaces can be achieved by relieving of complexity.

2) Direct and indirect adaptation: The direct and indirect adaptation can support to make an approach for a wider variety of scenarios. By providing alternative version of adapted UI or indirect adaptation, the confusion of users can be minimized.Yet the comparison between adaptive and adaptable interfaces is not mature enough, only in some systems (e.g. ubiquitous), it is necessary to adapt UI with direct adaptation. The study is conducted by developing prototype as a frontend to commercial word processor. For example, the participants used MSWord personal interface for evaluation in four weeks, where 14 out of 19 users spent almost less or more than $50 \%$ time in their personal interface usage [65]. In daily life, the smart environments which are being used in utilizing the computers as tools.It suppors the users for moving interaction 
(direct and indirect) with computers from a single system to a complex and distributed environment. The required synchronization [66] of the parts is a problem with distributed user interfaces (e.g. MASP) which adapts the smart home UIs on the basis of changability in environment.

3) Extensibility of adaptive behaviour: Extensibility is an important feature for the development of new user interface.It helps for multiple adaptation (e.g. accessibility, cognition etc.) of UI because it is not restricted to single type of adaptation such as layout optimization. The extensibility of adaptive behaviour makes available to add new adaptive behaviour at run time when desired to provision a diversity of aspects [67]. For example, COMET is modeled for supporting polymorphism that belongs to different technological spaces (e.g. HTML, OpenGL, vocal). The dynamic ability to tailortheuser interfaces with designer and end user to discover design alternatives by substituting COMET presentations at design as well as runtime.

4) Empowering new design participants: The new design participants can be non-developers (end users, IT personels) in the case of adaptive user interfaces. For example, leveraging communities through crowdsourcing could prove useful for applications that require a lot of effort for defining the adaptive behavior.

5) Integrated development environment: The integrated development environment (IDE) is a style of user interface which looks similar to Visual Studio or Eclips [68]. It can provide easiness for organization UI and adaptive behaviour articles of large scale software systems.

6) Supporting multiple levels of abstraction: The CAMELEONsuggeststhe liberty of task modeling, abstraction and technology of concrete UI. Moreover, the different levels might be appropriate for certain type of adaptation [67]. By adapting highest level, the UI features can be reduced and also through a number of levels, the layout could be optimized.

7) Selected modeling approach: In this approach the selected interpreted runtime modeling permits advanced adaptations to be conducted [69]. The old fashioned approach "model driven engineering" (MDE), used in HCI has revived conventional (WIMP) user interfaces [70]. This approach brings some hope by providing theoretical and task level modeling into a unifying and systematic approach to the problem of UI plasticity.

8) Modeling, generation, and synchronization: It provides abstraction at all levels where the tools of model driven UI development must create easiness to the developers. Predictability for the programmers is very important and should not be ignored during the development of tools [71]. However, synchronization is one of the main feature that creates user satisfaction.

9) Supporting multiple data sources: The architectures and procedures of adaptation enable the users interface generation and dynamic adaptivity during run time [11]. The multiple data sources permits adaptations to be carried out in various situations. The models having adaptive behaviour [72] can exemplify data on the basis of different studies, which is the case of adapting UIs to cultural preferences by MOCCA.

10) Preserving designer input on the UI: In some cases, [71] the designers may wish to preserve some characteristics to enhance the predictability of outcome.

11)Reducing solution viscosity: It is achieved if a tool reduces [68] the effort required to iterate on the possible solutions based on the [71] flexibility, express leverage, expressive match, scalability, low threshold, high ceiling andtrade off analysis.

12) User feedback on the adapted UI: The user feedback on AUI [72] provides awareness of automated adaptation decisions and the ability to take priority overthem whenever needed.

\section{Development Process Model of AUI}

This section discusses the development process model of adaptive user interface.

1) User center design process model: The philosophy of UCD is to analyze the usability and validity of mobile device interfaces according to user contexts. The AIO will provide the designing and scaling of mobile device interfaces according to the context of user. UCD is a framework of processes and methodology which is not restricted to interfaces or technologies [12]. It deals with the product for the understanding of needs, wants, tasks, environments, preferences and limitations in user's context and are given extensive attention at each stage of the design process. It is the process of designing software with interfaces and then solving of multi-stage problems from the perspective of user's understandability. The system can be designed for user's support with their existing beliefs, attitude and behaviors related to their tasks, rather than the users adapt or learn the designed system [73]. UCD approach is used to develop simple models, mock-ups or prototypes on parts or all of the designs such that graphical design, information architecture, interaction design, information visualization. The UCD not only requires designers to analyze and predict that how users use a product but also test the validity with regards to user's behavior. The testing of a product is necessary but it is difficult task for designers to understand the user's experiences. UCD has complete life-cycle to produce the products with high usability and low cost [4]. The major goal of UCD is to offer optimized, efficient and user friendly product which increases the usability and satisfaction of users.

2) Mapping UCD process model in UCO and AIO: All features of UCD will provide real benefits to the user experience. In UCD, the prototypes are very useful to translate the user requirements into contextual experience. It is used to enhance the usability, satisfaction and optimization of adaptive mobile user interfaces. UCO and AIO will be developed to optimize the use of adaptive mobile interfaces in the context of user preferences [74]. These ontologies organize the perceptions and thoughts of user. "Fig. 1" shows the proposed research roadmap for the engineering of UCO and AIO for 
mobile device interfaces in user's context. It also describes the mapping functions of UCD [50] with UCO to AIO which leads towards the experimentation and evaluation of results for the satisfaction of users.

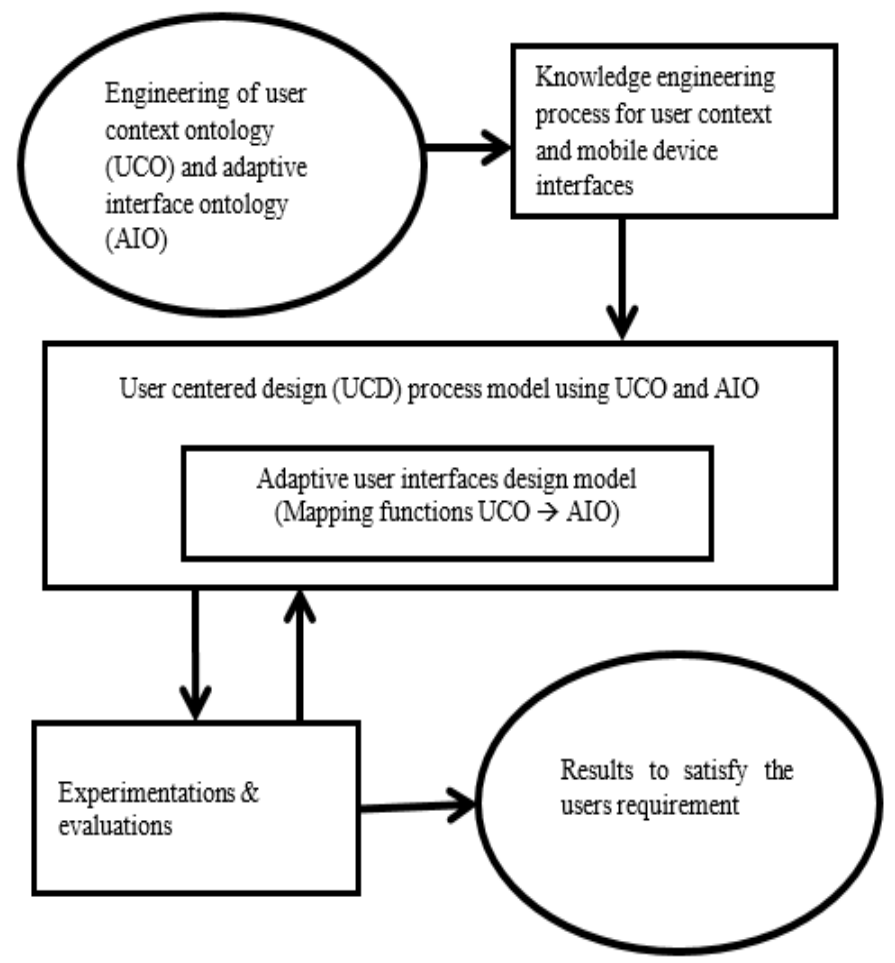

Fig. 1. Development Process Model for Adaptive Mobile User Interface.

\section{EVALUATION OF INTERFACE}

Usability attributes are classified in two types of measures, objective and subjective measures. There are five usability attributes that help us to evaluate an interface in human computer interaction. Objective measures are very useful in evaluation of an interface; however, the collection of data objectively is an expensive, time consuming and challenging task. On the other hand, subjective data can be collected easily, speedily and with comparatively less effort. Attributes like user opinion and preferences can also be measured using subjective approach of evaluation [75]. For instance, many of modern smart phone applications have multiple usability problems which include the navigation, poor support for performing tasks, complicated interfaces, complex interaction styles, limited interaction techniques and confusion due to a lot of options given to user. The magnitude of problem is increased for mobile phones because of their limited processing power, screen size, mobility and multiple network connectivity issues.

There exist a lot of algorithms that can be used to perform multiple types of adaptation and each algorithm has its strength in the evaluation process [76]. According to a study by Lewis in 2006, hidden smart menus of pre-2007 versions of Microsoft Office resulted in multiple usability issues, however, the revised version of MS Office released after 2007 contained predefined adaptive parts which used to show the most recently used tasks increased the user satisfaction and improved the overall usability [62], thus proving to be more beneficial for the user. Menthol project examined the parameters like age and gender on mobile phone usage and the results showed that these parameters have a direct relation to the usability of a smart phone. Due to the popularity and personal aspect, usability of a smartphone is one of the hot research areas in pervasive computing. The project analyzes the abovementioned factors where the dataset is an output of a longitudinal study. A sample of more than thirty thousand participants was selected, out of which around sixty thousand were male and fourteen thousand five hundred were female. The median age of the participants was 21 years and they were tracked for 28 days. The data of their personal and demographic details was submitted through a predefined questionnaire. The study was conducted in January 2014. It was observed that the average time a female spends on a smart phone is 166.78 minutes, whereas males spend 154.26 minutes, hence, females use their devices for a longer period then males. Another finding of the project was that the younger participants were more inclined towards entertainment and social networking applications through specialized software applications. Young users also spent more time then the users who are older in age [36]. After analysis it was found that the older users used the smart phones for getting general information and preferred to use the smart phone as a conventional phone.

In this paper we have analyzed the usability of the adaptive features that have been provided by the device manufacturers. The features that were analyzed for user satisfaction, efficiency and effectiveness. A user centric design was followed to evaluate the usability of features that are adaptive [4] [64]. It was seen that the users preferred the portrait mode while typing because it gives the user the ease of typing by using single hand, the overall effectiveness of the feature of screen rotation was $25 \%$ less effective. An interesting finding was that the voice command adaptive feature, despite of the fact that the feature was $6 \%$ more efficient, but surprisingly it was $19 \%$ less effective during the user interaction. One of the reasons that were observed in less effectiveness of the voice command feature was the problem in recognizing the accent of the user. The effectiveness of LED notifications adaptive feature was recorded as $88 \%$, not only this but the overall efficiency was also $89 \%$, hence, the LED notification feature was very effective and efficient. For kids the adaptive environment feature was found to be $28 \%$ more efficient and effective than using the smartphone in normal mode. It was observed that because of the identical adaptive features provided by multiple vendors, there were a lot of adaptability issues. Another point that creates the problems is that the vendors do not consider the user's ability to perform a task and the context of the task. From the experimental findings, it is inferred that the adaptivity has a positive impact on the usability of a smart phone, when it is applied in an appropriate context [17]. Another example that supports the findings was an adaptive bar which was presented by Debevc et al [77]. In this toolbar, the software system proposed the additions and deletions of tasks to the user; suggestions are given on the basis of history, frequency and context of the user. The results of another study show that the adaptive tool bar was more effective and helped users to build their own tool bar more efficiently, thus, enhancing the user experience. 


\section{CONCLUSION}

The fundamental purpose of HCI is to make the systems more usable, useful and to provide better usability according to user experiences. Many researchers have suggested AUI in their own specific perspective. Currently, the smart world has many variations in all aspects of user, task, environment and device. These deviations in usage environment are increasing speedily which cannot be handled by using single interface. Therefore, it is near to impossible to develop specialized interfaces for each context separately. In this survey paper, we studied different principles and mechanisms used in context models. User modelling research is discussed to address these types of issues. Likewise, semantic modelling has also provided a solution for complex scenarios and computational models. These complex contexts can be modelled by using semantics and ontologies. Furthermore, the user interface process model suggested development of AUIs on the basis of guidelines provided in this survey paper.

\section{REFERENCES}

[1] D. Benyon and D. Murray, "Applying user modelling to human-computer interaction design,” AI Rev., 1993.

[2] A. Petrovčič, A. Rogelj, and V. Dolničar, "Smart but not adapted enough: Heuristic evaluation of smartphone launchers with an adapted interface and assistive technologies for older adults," Computer Human Behaviour, 2018.

[3] R. Hervás and J. Bravo, "Towards the ubiquitous visualization: Adaptive user-interfaces based on the Semantic Web," Interact. Comput., 2011.

[4] M. W. Iqbal, N. Ahmad, and S. K. Shahzad, "Usability evaluation of adaptive features in smartphones," in Procedia Computer Science, 2017, vol. 112, pp. 2185-2194.

[5] P. A. Akiki, A. K. Bandara, and Y. Yu, "Adaptive Model-Driven User Interface Development Systems," ACM Comput. Surv., vol. 47, no. 1, pp. 1-33, 2014.

[6] H. Petrie and N. Bevan, "The evaluation of accessibility, usability and user experience," Univers. Access Handb., 2009.

[7] Ál. FernáNdez-LóPez, M. J. RodríGuez-FóRtiz, M. L. RodríGuezAlmendros, and M. J. MartíNez-Segura, "Mobile learning technology based on iOS devices to support students with special education needs," Comput. Educ., 2013.

[8] T. Paymans and J. Lindenberg, "Usability trade-offs for adaptive user interfaces: ease of use and learnability," Intell. user interfaces, no. c, pp. 1271-1274, 2004.

[9] S. Kane, "Improving Mobile Phone Accessibility with Adaptive User Interfaces," Ideals.Illinois.Edu, 2010.

[10] A. Ali, M. Alrasheedi, A. Ouda, and L. F. Capretz, "A Study of the Interface Usability Issues of Mobile Learning Applications for Smart Phones from the User's Perspective," Int. J. Integr. Technol. Educ., 2014.

[11] M. Peissner, D. Häbe, D. Janssen, and T. Sellner, "MyUI: Generating Accessible User Interfaces from Multimodal Design Patterns," in Proceedings of the 4th ACM SIGCHI Symposium on Engineering Interactive Computing Systems, 2012.

[12] S. D. I. Dottorato and N. Ahmad, "Tesi di Dottorato People Centered HMI's for Deaf and Functionally Illiterate Users," 2014.

[13] N. U. Bhaskar, S. N. Raju, G. Paladugu, and V. Reddy, "Aspects of Content, Context and Adaptation Modeling in Mobile Learning Application Design,” Int. J. Interact. Mob. Technol., vol. 7, no. 2, pp. 2937, 2013.

[14] F. Gulla, L. Cavalieri, S. Ceccacci, M. Germani, and R. Bevilacqua, Method to design adaptable and adaptive user interfaces, vol. 528. 2015.

[15] S. Greenberg and I. H. Witten, "Adaptive personalized interfaces: a question of viability,” Behav. Inf. Technol., 1985.

[16] a T. Submitted, I. N. Partial, F. Of, T. H. E. Requirements, F. O. R. The, and D. Of, "Comparing Static, Adaptable, and Adaptive Menus by The University of British Columbia," no. August, 2004.
[17] E. Tenner, "The Design of Everyday Things by Donald Norman," Technol. Cult., 2015.

[18] A. Cadenas et al., "Context Management in Mobile Environments: A Semantic Approach," Proc. 1st Work. Context. Inf. Ontol., p. 2:1--2:8, 2009.

[19] V. Glavinic, S. Ljubic, and M. Kukec, "Transformable Menu Component for Mobile Device Applications: Working with both Adaptive and Adaptable User Interfaces,” Int. J. Interact. Mob. Technol., 2008.

[20] R. Oppermann, Adaptive user support: ergonomic design of manually and automatically adaptable software. 1994.

[21] J. Fink, A. Kobsa, and A. Nill, "Adaptable and Adaptive Information Access for All Users, Including the Disabled and the Elderly BT-User Modeling," User Model., no. Chapter 19, pp. 171-173, 1997.

[22] L. Findlater and J. McGrenere, "A comparison of static, adaptive, and adaptable menus," Proc. ACM CHI 2004, vol. 6, no. 1, pp. 89-96, 2004.

[23] B. Shneiderman, "Direct manipulation for comprehensible, predictable and controllable user interfaces," Proc. 2nd Int. Conf. Intell. user interfaces- IUI '97, pp. 33-39, 1997.

[24] M. Manca and F. Paternò, "Customizable dynamic user interface distribution," in Proceedings of the 8th ACM SIGCHI Symposium on Engineering Interactive Computing Systems - EICS ’16, 2016.

[25] G. Fischer, "User modeling in human computer interaction," User Model. User Adapt. Interact., vol. 11, pp. 65-86, 2001.

[26] J. R. Lewis, "Usability Testing," Handbook of Human Factors Testing and Evaluation. pp. 1275-1316, 2006.

[27] J. Hewes, "Perspectives on Usability,” Conf. Rec. Crossing Front., 1992.

[28] N. A. Mian and F. Ahmad, "Modeling and Analysis of MAPE-K loop in Self Adaptive Systems using Petri Nets," vol. 17, no. 12, pp. 158-163, 2017.

[29] J. Nielsen, "Ten Usability Heuristics," Copyr. (c) 2005 by Jakob Nielsen. ISSN 1548-5552, pp. 1-2, 2005.

[30] R. Molich and J. S. Dumas, "Comparative usability evaluation (CUE-4)," Behav. Inf. Technol., 2008.

[31] R. Shehzad and N. Ahmad, "Web Usability and User Trust on Ecommerce Websites in Pakistan," vol. 8, no. 12, 2017.

[32] A. M. Figueroa, R. Juárez-Ramírez, S. Inzunza, and R. Valenzuela, "Implementing adaptive interfaces: A user model for the development of usability in interactive systems," Comput. Syst. Sci. Eng., 2014.

[33] J. Nielsen, "Finding usability problems through heuristic evaluation," Proc. SIGCHI Conf. Hum. factors Comput. Syst. - CHI '92, pp. 373380, 1992.

[34] R. Molich et al., "Comparative evaluation of usability tests," CHI'99 Ext.vol. 2, no. May, p. 83, 1999.

[35] ISO, "Iso 9241-11," Ergon. Requir. Off. Work with Vis. Disp. Termin. Part 11 Guid. usability, vol. 1998, p. 22, 1998.

[36] I. Andone, K. Błaszkiewicz, M. Eibes, B. Trendafilov, C. Montag, and A. Markowetz, "How age and gender affect smartphone usage," in Proceedings of the 2016 ACM International Joint Conference on Pervasive and Ubiquitous Computing Adjunct - UbiComp '16, 2016.

[37] G. Jindal and M. Jain, "A Comparative Study of Mobile Phone's Operating Systems,” Int. J. Comput. Appl. Inf. Technol. I, Issue III, vol. 1, no. 3, pp. 2278-7720, 2012.

[38] N. Ahmad, M. W. Boota, and A. H. Masoom, "Smart Phone Application Evaluation with Usability Testing Approach," J. Softw. Eng. Appl., vol. 07, no. 12, p. 1045, 2014.

[39] J. Eisenstein, J. Vanderdonckt, and A. Puerta, "Adapting to mobile contexts with user-interface modeling," Proc. - 3rd IEEE Work. Mob. Comput. Syst. Appl. WMCSA 2000, pp. 83-92, 2000.

[40] I. Sciences, "Transparency in Adaptive Mobile User Interfaces Gaël van Heijst," no. June, 2016.

[41] A. K. Dey and G. D. Abowd, "Towards a Better Understanding of Context and Context-Awareness,' Comput. Syst., vol. 40, no. 3, pp. 304307, 1999.

[42] M. Zheng, S. Cheng, and Q. Xu, "Context-Based Mobile User Interface," no. July, pp. 1-9, 2016. 
[43] I. Uddin, A. Rakib, H. M. U. Haque, and P. C. Vinh, "Modeling and Reasoning about Preference-Based Context-Aware Agents over Heterogeneous Knowledge Sources," Mob. Networks Appl., vol. 23, no. 1, pp. 13-26, 2018.

[44] J. Park and S. H. Han, "Integration of Adaptable and Adaptive Approaches for Interface Personalization Through Collaborative Menu," Int. J. Hum. Comput. Interact., vol. 28, no. 9, pp. 613-626, 2012.

[45] R. Mizouni, M. A. Matar, Z. Al Mahmoud, S. Alzahmi, and A. Salah, "A framework for context-aware self-adaptive mobile applications SPL," Expert Syst. Appl., vol. 41, no. 16, pp. 7549-7564, 2014.

[46] S. A. Hoseini-Tabatabaei, A. Gluhak, and R. Tafazolli, "A survey on smartphone-based systems for opportunistic user context recognition," ACM Comput. Surv., vol. 45, no. 3, pp. 1-51, 2013.

[47] N. Bevan and M. Macleod, "Usability measurement in context," Behav. Inf. Technol., vol. 13, no. 1-2, pp. 132-145, 1994.

[48] M. Zheng, O. Ormandjieva, and H. Fan, "Designing Context Sensitive Mobile User Interface," in Proceedings of the International Conference on Software Engineering Research and Practice (SERP), 2015.

[49] A. Schmidt, M. Beigl, and H. W. Gellersen, "There is more to context than location," Comput. Graph., vol. 23, no. 6, pp. 893-901, 1999.

[50] S. K. Shahzad, "Ontology-based User Interface Development: User Experience Elements Pattern,” J. Univers. Comput. Sci., vol. 17, no. 7, pp. 1078-1088, 2011.

[51] K. L. Skillen, L. Chen, C. D. Nugent, M. P. Donnelly, and I. Solheim, “A user profile ontology based approach for assisting people with dementia in mobile environments.," Conf. Proc. IEEE Eng. Med. Biol. Soc., vol. 2012, pp. 6390-3, 2012.

[52] J. Hussain et al., "Model-based adaptive user interface based on context and user experience evaluation," J. Multimodal User Interfaces, vol. 12, no. 1, pp. 1-16, 2018.

[53] V. Wiens and S. Lohmann, "Device-Independent Visual Ontology Modeling," 2018, pp. 1-15.

[54] M. Poveda-Villalón, M. C. Suárez-Figueroa, R. García-Castro, and A. Gómez-Pérez, "A context ontology for mobile environments," in CEUR Workshop Proceedings, 2010, vol. 626.

[55] M. Baldauf, S. Dustdar, and F. Rosenberg, "A survey on context-aware systems,” Int. J. Ad Hoc Ubiquitous Comput., vol. 2, no. 4, p. 263, 2007.

[56] G. Meditskos, S. Dasiopoulou, and I. Kompatsiaris, "MetaQ: A knowledge-driven framework for context-aware activity recognition combining SPARQL and OWL 2 activity patterns," Pervasive Mob. Comput., vol. 25, pp. 104-124, 2016.

[57] M. W. Iqbal and N. Ahmad, "Adaptive Interface for Color-Blind People in Mobile-Phones," 2018.

[58] T. Lavie and J. Meyer, "Benefits and costs of adaptive user interfaces," Int. J. Hum. Comput. Stud., vol. 68, no. 8, pp. 508-524, 2010.

[59] A. Holzinger, M. Geier, and P. Germanakos, "On the development of smart adaptive user interfaces for mobile e-business applications: Towards enhancing user experience - Some lessons learned," in 3rd International Conference on Data Communication Networking, DCNET 2012.
[60] I. Riahi and F. Moussa, "A formal approach for modeling context-aware Human-Computer System,” Comput. Electr. Eng., vol. 44, pp. 241-261, 2015.

[61] R. Klaassen, R. op den Akker, T. Lavrysen, and S. van Wissen, "User preferences for multi-device context-aware feedback in a digital coaching system,” J. Multimodal User Interfaces, vol. 7, no. 3, pp. 247-267, 2013.

[62] M. Hartmann, "Challenges in Developing User-Adaptive Intelligent User Interfaces," Proc. 17th Work. Adapt. User Model. Interact. Syst., pp. 6$11,2009$.

[63] K. Olga, "Adaptive User Interface Patterns for Mobile Applications,"2014.

[64] N. A. Mian and F. Ahmad, "Modeling and Analysis of MAPE-K loop in Self Adaptive Systems using Petri Nets," vol. 17, no. 12, pp. 158-163, 2017.

[65] J. Mcgrenere, R. M. Baecker, and K. S. Booth, "An evaluation of a multiple interface design solution for bloated softwarelproceedings of the sigchi conference on human factors in computing systems: Changing our world, changing ourselves," Chi '02, no. 4, pp. 164-170, 2002.

[66] M. Blumendorf and S. Feuerstack, "Event-based Synchronization of Model- Based Multimodal User Interfaces," 2006.

[67] A. Demeure, G. Calvary, and K. Coninx, "COMET(s), A Software Architecture Style and an Interactors Toolkit for Plastic User Interfaces," in Lecture Notes in Computer Science, 2008.

[68] D. R. Olsen Jr., "Evaluating user interface systems research," 20th Annu. ACM Symp. User interface Softw. Technol., pp. 251-258, 2007.

[69] R. Soley and OMG Staff Strategy Gropu, "Model driven architecture," OMG white Pap., no. April, pp. 1-12, 2000.

[70] J. Coutaz, "User Interface Plasticity: Model Driven Engineering to the Limit!” Proc. 2Nd ACM SIGCHI Symp. Eng. Interact. Comput. Syst., 2010.

[71] B. Myers, S. E. Hudson, and R. Pausch, "Past, present, and future of user interface software tools," ACM Trans. Comput. Interact., vol. 7, no. 1, pp. $3-28,2000$.

[72] P. A. Akiki, "Engineering adaptive user interfaces for enterprise applications," EICS 2013 - Proc. ACM SIGCHI Symp. Eng. Interact. Comput. Syst., pp. 151-154, 2013.

[73] R. Roth, K. Ross, and A. MacEachren, "User-Centered Design for Interactive Maps: A Case Study in Crime Analysis," ISPRS Int. J. GeoInformation, vol. 4, no. 1, pp. 262-301, 2015.

[74] E. Castillejo, A. Almeida, and D. López-de-Ipiña, "Ontology-Based Model for Supporting Dynamic and Adaptive User Interfaces," Int. J. Hum. Comput. Interact., 2014.

[75] A. Hussain and U. Utara, "Apps vs Devices: Can the Usability of Mobile Apps can be Decoupled from the Device? Apps vs Devices: vol. 9, no. May 2016, pp. 11-16, 2012.

[76] J. L. Wesson et al., "Can Adaptive Interfaces Improve the Usability of Mobile Applications? 2014.

[77] M. Debevc, B. Meyer, D. Donlagic, and R. Svecko, "Design and evaluation of an adaptive icon toolbar," User Modeling and User-Adapted Interaction. 1996. 